\title{
Slowing of Bessel light beam group velocity
}

\author{
Robert R. Alfano ${ }^{1,3^{*}}$ and Daniel A. Nolan ${ }^{2,3}$ \\ ${ }^{I}$ Physics Department, City College of New York of the City University of New York, \\ 160 Convent Ave. New York, NY 10031 USA \\ ${ }^{2}$ Corning Incorporated, Sullivan Park, Corning, NY 14830 USA \\ ${ }^{3}$ Center for Complex Light, CCNY, New York, NY 10031 USA \\ *Corresponding author: ralfano@sci.ccny.cuny.edu
}

Received Month X, XXXX; revised Month X, XXXX; accepted Month X, XXXX; posted Month X, XXXX (Doc. ID XXXXX); published Month X, XXXX

\begin{abstract}
Bessel light beams experience diffraction-limited propagation. A different basic spatial property of a Bessel beam is reported and investigated. It is shown a Bessel beam is a natural waveguide causing its group velocity can be subluminal (slower than the speed of light) when the optical frequency $w$ approaches a critical frequency $w_{\mathrm{c}}$. A free space dispersion relation for a Bessel beam, the dependence of its wave number on its angular frequency, is developed from which the Bessel beam's subluminal group velocity is derived. It is shown under reasonable laboratory conditions that a Bessel light beam has associated parameters that allow slowing near a critical frequency. The application of Bessel beams with 1 um spot size to slow down 100 ps to 200 ps over $1 \mathrm{~cm}$ length for a natural optical buffer in free space is presented.

OCIS Codes: (080.4865) Optical vortices, (080.1510) Propagation methods, (200.4490) Optical buffers..

http://dx.doi.org/10.1364/OL.99.099999
\end{abstract}

Light's salient degrees of freedom are the independent parameters that completely describe an electromagnetic wave (in the paraxial approximation) and include polarization, wavelength, and time [1]. Most recently, light's space degree of freedom has received significant attention via the sub-discipline of optics that can be referred to as complex light or structured light [2]. The study of complex light is a veritable renaissance of optics; using light's space degree of freedom many classical optics phenomena have been revisited with novel results, such as double slit diffraction [3], the mechanical Faraday effect [4,5], and Fermat's principle [6]. Additionally, using light's space degree of freedom the fundamental limits of many optics applications have been addressed, e.g., the transmission data rate of optical fiber communication can potentially be increased beyond that single mode optical fibers via space division multiplexing $[7,8]$, and it is possible to image below the diffraction limit via STED microscopy $[9,10]$.

A Bessel light beam is an example of a light beam described by a special function. A Bessel beam is a light beam that in contrast to more conventional Gaussian beams possesses noteworthy properties, such as, "self-healing" and diffraction-limited propagation associated with a pencil-like beam profile [11, 12]. Due to these properties, Bessel beams have been extensively studied and used for a number of applications; for comprehensive reviews see $[13,14]$. When using a Bessel beam for optical trapping it is possible to simultaneously trap multiple particles in well-separated planes [15], and make a particle tractor beam [16, 17]. Also, when using a Bessel beam's line profile for light sheet microscopy it is possible to rapidly image biological samples (e.g. tissue) in three-dimensions [18, 19].

In this work, the dispersion of a Bessel beam in free space is investigated. It is shown a Bessel beam is a natural waveguide and its group velocity can be subluminal (slower than the speed of light) as compared to a Gaussian beam in free space. A free space dispersion relation for a Bessel beam, i.e., the dependence of its wave number on its angular frequency, is outlined from which the Bessel beam's subliminal group velocity is derived. It is shown for reasonable laboratory conditions a Bessel light beam has associated parameters that allow slowing near a critical frequency. The application of Bessel beams for a natural optical buffer in free space is presented. is $[11,12]$ :

The wave equation in cylindrical coordinates

$$
\left(\nabla^{2}(r,, z) k^{2}\right) \quad(r,, z)=0,
$$

where $(r,, z)$ are cylindrical coordinates, $k=2$ / is the wave number, is light's wavelength. As a solution to the wave equation a Bessel beam is derived via separation of variables where $(r,, z)=R(r) \quad() Z(z)$ :

$$
(r, \quad, z)=J_{o}(k r) \exp \left(i k_{z} z\right) \exp \left(\begin{array}{ll}
i & t
\end{array}\right)
$$

where $k$ and $k_{z}$ are the transverse and axial 
propagation wave numbers, respectively.

The relationship between the transverse and axial wave numbers is:

$$
k^{2}(\quad)=k^{2}+k_{z}^{2}(\quad) \text {. }
$$

The propagation constant of the beam is given by dispersion relationship:

$$
k()=\frac{n(\quad)}{c}
$$

where $n(\quad)$ is the index of refraction of the medium in which the Bessel light beam propagates, $c$ is the speed of light, and is light's angular frequency.

Using Eq. 4, Eq. 3 can be rewritten as dispersion relation:

$$
\begin{aligned}
& \omega^{2}\left(k_{z}\right)=\frac{c^{2}}{n^{2}} k_{z}^{2}+\frac{c^{2}}{n^{2}} k_{\perp}^{2}, \\
& \omega\left(k_{z}\right)=\left(\frac{c^{2}}{n^{2}} k_{z}^{2}+\frac{c^{2}}{n^{2}} k_{\perp}^{2}\right)^{1 / 2},
\end{aligned}
$$

and

$$
\left(k_{z}\right)=\frac{c^{2}}{n^{2}} k_{z}^{2}+{ }_{c}^{2} \div \text {. }
$$

The critical frequency is defined as:

$$
\omega_{c} \equiv \frac{c k_{\perp}}{n}
$$

which represents the frequency of the standing waves in perpendicular transverse direction.

Effectively, Eq. 7 embodies a free space dispersion relationship $\omega$ vs $k$ for a Bessel light beam in free space, i.e., the dependence of its angular frequency on its axial wave vector.

The group velocity of a Bessel light beam can then be derived from this dispersion relation via the definition of group velocity [1]:

$$
v_{g, z}=\frac{\left(k_{z}\right)}{k_{z}} \text {. }
$$

Subsequently $v_{g}$ becomes

$$
v_{g, z}=\frac{1}{2} \frac{c^{2}}{n^{2}} 2 k_{z} \frac{c^{2}}{n^{2}} k_{z}^{2}+{ }_{c}^{2} \div,
$$

and

$$
v_{g, z}=\frac{\frac{c^{2}}{n^{2}} k_{z}}{\frac{c^{2}}{n^{2}} k_{z}^{2}+{ }_{c}^{2} \div} .
$$

Using Eq. 3, Eq. 11 can be rewritten as:

$$
v_{g, z}=\frac{\frac{c^{2}}{n^{2}}\left(k^{2} k^{2}\right)}{\frac{c^{2}}{n^{2}} k^{2} \frac{c^{2}}{n^{2}} k^{2}+{ }_{c}^{2} \div} .
$$

Using Eq. 8, Eq. 12 can be rewritten as:

$$
v_{g, z}=\frac{\frac{c^{2}}{n^{2}}\left(k^{2} k^{2}\right)^{1 / 2}}{\left(k^{2}\right)^{1 / 2}} .
$$

Finally, using Eq. 4 and again Eq. 8, Eq. 13 can be rewritten for $v_{g, z}$ as:

$$
v_{g, z}=\frac{c}{n}\left(1-\left(\frac{\omega_{c}}{\omega}\right)^{2}\right)^{1 / 2} .
$$

From Eq. 14, when $\omega$ approaches the critical frequency $v_{g, z}=0$ the Bessel beam stops. Fig. 1a shows $v_{g, z}$ vs $\omega$. For $\left(\omega=\omega_{c}\right)$, the beam is frozen.

The critical frequency is defined via the transverse wave number and diameter of the Bessel light beam to form a set of standing waves

$$
k=\frac{2}{a}
$$

where $a$ is the beam diameter of the central lope Bessel light beam. Consider a Bessel light beam with a beam diameter of $a=.63 \mathrm{~m}$ propagating in air $(n=1)$. The corresponding transverse wave number is given by $k \quad 10 \mathrm{~m}^{1}$. Then, the Bessel light beam's critical frequency is given by ${ }_{c}=3 f_{S}{ }^{1}$ corresponding to a wavelength of ${ }_{c} 632 n \mathrm{~m}$ (red light). 
A plot of $v_{g, z}$ (Eq. 14) for the example just described is shown in Fig. 1(a). Slowing occurs near $c^{\cdot}$

An effective group index of refraction $n_{g}$ can also be defined from Eq. 14:

$$
n_{g}=\frac{c}{v_{g, z}},
$$

and

$$
n_{g}=n\left(1-\left(\frac{\omega_{c}}{\omega}\right)^{2}\right)^{-1 / 2} .
$$

A plot of the group index $n_{g}$ (Eq. 17) for the same example shown in Fig. 1(b). The group index $n_{g}$ becomes large as $\omega \rightarrow \omega_{c}$; at $\omega=\omega_{c}, n_{g} \rightarrow \infty$.

The natural slowing of a Bessel light beam in free space can be used as a free space delay line or an optical buffer. As examples, consider Bessel light beams whose wavelengths are given by $632 \mathrm{~nm}$ and $800 \mathrm{~nm}$. The parameters for $632 \mathrm{~nm}$ become $k=2 / a, a() 0.63$, and $k=10 \mathrm{~m}$

${ }^{1}$ for $n=1$ (air) such that ${ }_{c}=k c / n=3 f s^{1}$. The laser $\quad 632 \mathrm{~nm}, \quad k \quad 2.98 f_{s}{ }^{1}$, slowing will occur. The parameters for $800 \mathrm{~nm}$ become $k=2 / a$, $a\left(\right.$ ) 1 , and $k=6.3 \mathrm{~m}^{1}$ for $n=1$ (air) such that ${ }_{c}=k c / n=2.4 f_{S}{ }^{1}$ and laser ${ }_{L}=2.4 f_{S}{ }^{1}$ will result in $v_{g} \quad 0$.

Consider the time it takes that Bessel light beam propagate over a distance $\ell$ given by $t=\ell / v_{g}$ as compared to a Gaussian light beam propagating over the same distance using an axicon lens or SLM over beam length of $\ell=1 \mathrm{~cm}$ and beam waist of a $1 \mathrm{~m}$. For $n_{\mathrm{g}} \sim 3$ the time delay is given by $100 \mathrm{ps}$ to $200 \mathrm{ps}$.

In support of Bessel beam slow down concept in free space and in dielectric media with index $\mathrm{n}$, Padgett reported in Science [20] and most recently at ICOAM conference $8 / 4 / 15$ on experimentally measuring propagation delay of Bessel beam on the order of few 10's fs. The salient difference of our research from [20] is that we show dependence of Bessel beam slow down on transverse beam size, a, the value of critical angular frequency $\omega_{c}$ near input laser frequency $\omega$, and dependence of group index $n_{g}$ on Bessel carrier angular frequency, $\omega$. In addition, selecting Bessel beam spot size of a around $1 \mu \mathrm{m}$ the beam can be used as an optical buffer to obtain 100 ps delay. Finally, the concept of Bessel beam being a natural waveguide for slowing light was submitted as a disclosure for patenting in November 2011 and delayed this submission.

In conclusion, it was shown a Bessel light beam is a natural waveguide in air and in dielectric. Its group velocity can be subluminal (slower than the speed of light). A free space dispersion relation for a Bessel light beam, i.e., the dependence of its wave number on its angular frequency, was outlined from which the Bessel beam's subluminal group velocity was derived. It was shown for reasonable laboratory conditions a Bessel has associated parameters that allow slowing near a critical frequency. The application of Bessel beams for a natural optical buffer in free space is described.

We acknowledge support from ARO Grant No. 47221-00-01 and Corning, Inc. We thank G. Milione for help in preparing the manuscript and early discussions.

References.

[1] M. Born and E. Wolf, Principles of optics: electromagnetic theory of propagation, interference, and diffraction, (Cambridge University Press, Cambridge, 1999).

[2] D. L. Andrews, Structured Light and its Applications (Academic Press, New York, 2008).

[3] H. I . Sztul and R. R. Alfano, Opt. Lett. 31, 999-1001 (2006).

[4] M. J. Padgett, G. Whyte, J. Girkin, A. Wright, L. Allen, P. Ohberg, and S. Barnett, Opt. Lett. 31, 2205-2207 (2006).

[5] S. F. Ranke-Arnold, G. Gibson, R. W. Boyd, M. J. Padgett, Science 333, 65 (2011).

[6] N. Yu, P. Genevet, M. A. Kats, F. Aieta, J. P. Tetienne, F. Capasso, and Z. Gaburro, Science 334, 333 (2011).

[7] D. J. Richardson, J. M. Fini, and L. E. Nelson, Nat. Photonics 7(5), 354362 (2013).

[8] G. Li, N. Bai, N. Zhao, and C. Xia, Adv. Opt. Photon. 6, 413487 (2014).

[9] S. W. Hell, Science 316, 1153-1158 (2007).

[10] S. W. Hell and J. Wichmann, Opt. Lett. 19, 780-782 (1994).

[11] J. Durnin, J. Opt. Soc. Am. A 4, 651 (1987).

[12] J. Durnin, J. J. Miceli, Jr., and J. H. Eberly, Phys. Rev. Lett. 58, 1499 (1987).

[13] M. Mazilu, J.D. Stevenson, F. Gunn-Moore, and K. Dholakia, Laser and Photon. Rev. 4, 529 (2010).

[14] D. Mcgloin and K. Dholakia, Contemp. Phys. 46,15 (2005).

[15] V. Garcs-Chvez, D. McGloin, H. Melville, W. Sibbett, and K. Dholakia, Nature 419, 145-147 (2002).

[16] S. Sukhov and A. Dogariu, Phys. Rev. Lett. 107, 203602 (2011).

[17] D. B. Ruffner and D. G. Grier, Phys. Rev. Lett. 109, 163903 (2012).

[18] T. A. Planchon, L. Gao, D. E. Milkie, M. W. Davidson, J. A. Galbraith, C. G. Galbraith, and E. Betzig, Nature Methods 8, 417- 423 (2011).

[19] F. O. Fahrbach, P. Simon, and A. Rohrbach, Nat. Photonics 4(11), 780785 (2010)

[20] D. Giovannini, J. Romero, V. Potoček, G. Ferenczi, F. Speirits, S. M. Barnett, D. Faccio, M. J. Padgett, Science 347, 857, (2015). 
(a)

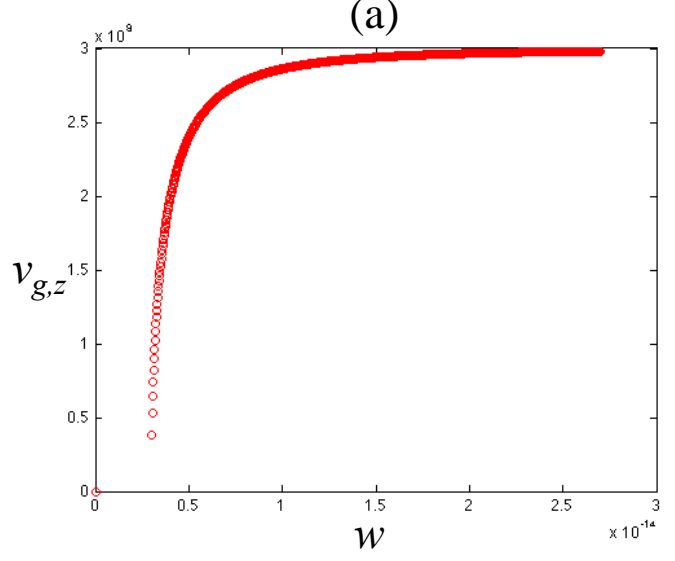

(b)

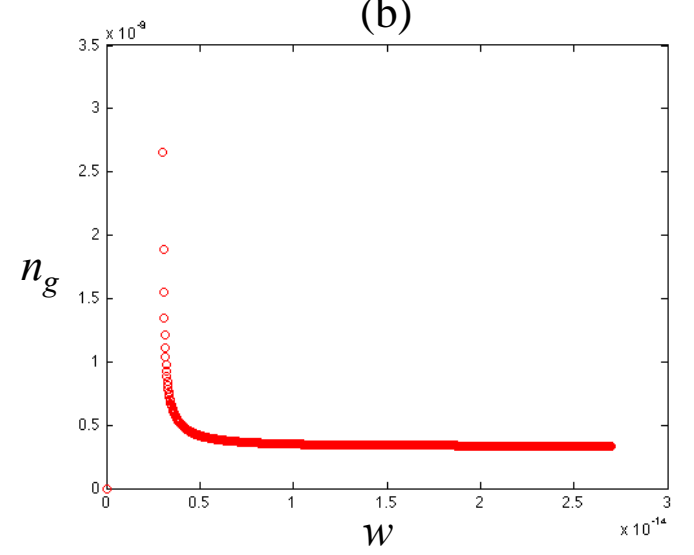

Fig. 1. (a) Group velocity of a Bessel light beam as a function of angular frequency (b) Group index of a Bessel light beam as a function of angular frequency. 